\title{
An Extension of the Hille-Hardy Formula*
}

\author{
By H. M. Srivastava
}

Abstract. While attempting to give extensions of the well-known Hille-Hardy formula for the generalized Laguerre polynomials $\left\{L_{n}^{(\alpha)}(x)\right\}$ defined by

$$
(1-t)^{-1-\alpha} \exp \left[-\frac{x t}{1-t}\right]=\sum_{n=0}^{\infty} L_{n}^{(\alpha)}(x) t^{n},
$$

the author applies here certain operational techniques and the method of finite mathematical induction to derive several bilinear generating functions associated with various classes of generalized hypergeometric polynomials.

It is observed that the earlier works of Brafman [2], [3], [4], Chaundy [5], Meixner [12], Weisner [16], and others quoted in the literature, are only specialized or limiting forms of the results presented here.

1. Introduction. In the course of an attempt to give extensions of the wellknown Hille-Hardy formula** (cf., for instance, [8, p. 189])

$$
\begin{aligned}
& \sum_{n=0}^{\infty} \frac{n !}{\Gamma(n+\alpha+1)} L_{n}{ }^{(\alpha)}(x) L_{n}{ }^{(\alpha)}(y) z^{n} \\
& \quad=(1-z)^{-1} \exp \left[-\frac{(x+y) z}{1-z}\right](x y z)^{-\alpha / 2} I_{\alpha}\left[\frac{2(x y z)^{1 / 2}}{1-z}\right], \quad|z|<1,
\end{aligned}
$$

associated with the Laguerre polynomial

$$
L_{n}^{(\alpha)}(z)=\sum_{k=0}^{n}\left(\begin{array}{l}
n+\alpha \\
n-k
\end{array}\right) \frac{(-z)^{k}}{k !}
$$

we invoke here the Laplace and the inverse Laplace transform techniques and the method of finite mathematical induction to obtain several bilinear generating functions for certain classes of generalized hypergeometric polynomials. Various specialized and limiting cases of our formulas appear in the earlier works of Brafman [2], [3], [4], Chaundy [5], Meixner [12], Weisner [16], and others quoted in the literature.

2. The Bilinear Generating Functions. In the binomial expansion

$$
(1-z)^{-\lambda}=\sum_{n=0}^{\infty} \frac{(\lambda)_{n}}{n !} z^{n}, \quad|z|<1,
$$

if we replace $z$ by $z(1-x /(p-\xi))$ and multiply both sides by $(p-\xi)^{-\alpha-1}$, we obtain

Received August 21, 1968.

* Notices Amer. Math. Soc., v. 15, 1968, pp. 634-635, Abstract 68T-449.

** For the history of the Hille-Hardy formula see Hardy [10, p. 192] and Watson [15, p. 190]. See also Hille [11]. 


$$
\begin{aligned}
(1-z)^{-\lambda} \frac{1}{(p-\xi)^{\alpha+1}}(1 & \left.+\frac{X}{p-\xi}\right)^{-\lambda} \\
& =\sum_{n=0}^{\infty} \frac{(\lambda)_{n}}{n !} z^{n} \frac{(p-x-\xi)^{n}}{(p-\xi)^{\alpha+n+1}},
\end{aligned}
$$

where $\operatorname{Re}(x)>0, \operatorname{Re}(p-\xi)>0$, and $X=x z /(1-z)$.

We now take the inverse Laplace transforms of both sides of (2.1), using the known operational relations (1), p. 217, and (31), p. 175 in [9], and we arrive at the familiar generating function $[6$, p. $654(5,7)]$

$$
(1-z)^{-\lambda}{ }_{1} F_{1}\left[\begin{array}{c}
\lambda \\
1+\alpha ;
\end{array}-\frac{x z}{1-z}\right]=\sum_{n=0}^{\infty} \frac{(\lambda)_{n}}{(1+\alpha)_{n}} L_{n}{ }^{(\alpha)}(x) z^{n}
$$

valid, by analytic continuation, for $|z|<1$.

Next we replace $z$ by $z(1-y /(p-\xi))$, multiply both sides by $(p-\xi)^{-\beta-1}$ and take their inverse Laplace transforms, and from (2.2) we similarly obtain

$$
\begin{aligned}
& \sum_{n=0}^{\infty} n ! \frac{(\lambda)_{n}}{(1+\alpha)_{n}(1+\beta)_{n}} L_{n}{ }^{(\alpha)}(x) L_{n}{ }^{(\beta)}(y) z^{n}=(1-z)^{-\lambda} \exp \left[-\frac{x z}{1-z}\right] \\
& \cdot \Phi^{(3)}\left[1-\lambda+\alpha, \lambda ; 1+\alpha, 1+\beta ; \frac{x z}{1-z}, \frac{y z}{z-1}, \frac{x y z}{(1-z)^{2}}\right], \quad|z|<1,
\end{aligned}
$$

where

$$
\Phi^{(3)}[\alpha, \beta ; \gamma, \delta ; x, y, z]=\sum_{m, n, p=0}^{\infty} \frac{(\alpha)_{m}(\beta)_{n+p}}{(\gamma)_{m+p}(\delta)_{n+p}} \frac{x^{m}}{m !} \frac{y^{n}}{n !} \frac{z^{p}}{p !}
$$

The formula (2.3), also proved by Erdélyi (see, e.g., [7, p. 288 (27)]) in a different form, may be looked upon as a 'mild' extension of the Hille-Hardy formula (1.1) to which it would obviously reduce when $\lambda \rightarrow 1+\alpha$ and $\alpha=\beta$.

In view of (2.4), the formula (2.3) can be written as

$$
\begin{aligned}
& \sum_{n=0}^{\infty} n ! \frac{(\lambda)_{n}}{(1+\alpha)_{n}(1+\beta)_{n}} L_{n}^{(\alpha)}(x) L_{n}{ }^{(\beta)}(y) z^{n} \\
&=(1-z)^{-\lambda} \exp \left[-\frac{x z}{1-z}\right] \sum_{n=0}^{\infty} \frac{(\lambda)_{n}}{n !(1+\beta)_{n}}\left(\frac{y z}{z-1}\right)^{n} \\
& \cdot \Phi_{2}\left[-n, 1-\lambda+\alpha ; 1+\alpha ; \frac{x}{1-z}, \frac{x z}{1-z}\right], \quad|z|<1,
\end{aligned}
$$

where $\Phi_{2}$ denotes the Humbert function [7, p. 225]

$$
\Phi_{2}[\alpha, \beta ; \gamma ; x, y]=\sum_{m, n=0}^{\infty} \frac{(\alpha)_{m}(\beta)_{n}}{(\gamma)_{m+n}} \frac{x^{m}}{m !} \frac{y^{n}}{n !} .
$$

Replace $x$ in (2.5) by $x t$, multiply both sides by $t^{\mu-1}$ and take their Laplace transforms with respect to $t$ using the known results (33), p. 191 in [8] and (4), p. 222 in [9]. A similar operation on the variable $y$ will finally give us 


$$
\begin{aligned}
\sum_{n=0}^{\infty} \frac{(\lambda)_{n}}{n !}{ }_{2} F_{1}\left[\begin{array}{l}
-n, \mu ; \\
1+\alpha ;
\end{array}\right]{ }_{2} F_{1}\left[\begin{array}{l}
-n, \nu ; \\
1+\beta ;
\end{array}\right] z^{n} \\
=(1-z)^{\mu-\lambda}(1-z+z x)^{-\mu}{ }_{n=0}^{\infty} \frac{(\lambda)_{n}(\nu)_{n}}{n !(1+\beta)_{n}}\left(\frac{y z}{z-1}\right)^{n} \\
\cdot F_{1}\left[\mu,-n, 1-\lambda+\alpha ; 1+\alpha ; \frac{x}{1-z+z x}, \frac{x z}{1-z+z x}\right]
\end{aligned}
$$

in terms of the Appell function [7, p. 224]

$$
F_{1}\left[\alpha, \beta, \beta^{\prime} ; \gamma ; x, y\right]=\sum_{m, n=0}^{\infty} \frac{(\alpha)_{m+n}(\beta)_{m}\left(\beta^{\prime}\right)_{n}}{(\gamma)_{m+n}} \frac{x^{m}}{m !} \frac{y^{n}}{n !} .
$$

The form of (2.6) suggests the existence of the more general result

$$
\begin{gathered}
\sum_{n=0}^{\infty} \frac{(\lambda)_{n}}{n !}{ }_{2} F_{1}\left[\begin{array}{c}
-n, \mu ; x \\
\nu ;
\end{array}{ }_{r+1} F_{s}\left[\begin{array}{r}
-n, \alpha_{1}, \cdots, \alpha_{r} ; y \\
\beta_{1}, \cdots, \beta_{s} ;
\end{array}\right] z^{n}\right. \\
=(1-z)^{\mu-\lambda}(1-z+z x)^{-\mu} \sum_{n=0}^{\infty} \frac{(\lambda)_{n}\left(\alpha_{1}\right)_{n} \cdots\left(\alpha_{r}\right)_{n}}{n !\left(\beta_{1}\right)_{n} \cdots\left(\beta_{s}\right)_{n}}\left(\frac{y z}{z-1}\right)^{n} \\
\cdot F_{1}\left[\mu,-n, \nu-\lambda ; \nu ; \frac{x}{1-z+z x}, \frac{x z}{1-z+z x}\right],
\end{gathered}
$$

provided $r \leqq s$ and $|z|$ is sufficiently small.

Indeed the formula (2.7) holds for $r=s=1$ by virtue of (2.6). For a proof by the method of finite mathematical induction, let us assume that it remains true for some values of $r$ and $s$, replace $y$ by $y t$, multiply both sides by $t^{\alpha_{r+1}-1}$, and take their Laplace transforms with respect to $t$. Then using the known integral (17), p. 219 in [9], we find that $r$ is replaced by $r+1$, thus completing the induction on $r$. To effect the induction on $s$, replace $y$ by $y / t$, multiply both sides by $t^{\boldsymbol{\beta}_{s+1}}$, and take their inverse Laplace transforms using the operational relation (1), p. 297 in [9].

Similar are the proofs of the following bilinear generating functions:

$$
\begin{aligned}
& \sum_{n=0}^{\infty} \frac{(\lambda)_{n}}{n !}{ }_{2} F_{1}\left[\begin{array}{r}
-n, \mu ; \\
\nu
\end{array} ;{ }_{r+2} F_{s}\left[\begin{array}{r}
-n, \lambda+n, \alpha_{1}, \cdots, \alpha_{r} ; \\
\beta_{1}, \cdots, \beta_{s} ;
\end{array}\right] z^{n}\right. \\
& =(1-z)^{\mu-\lambda}(1-z+z x)^{-\mu} \sum_{n=0}^{\infty} \frac{(\lambda)_{2 n}\left(\alpha_{1}\right)_{n} \cdots\left(\alpha_{r}\right)_{n}}{n !\left(\beta_{1}\right)_{n} \cdots\left(\beta_{s}\right)_{n}}\left[-\frac{y z}{(1-z)^{2}}\right]^{n} \\
& \text { - } F_{1}\left[\mu,-n, \nu-\lambda-n ; \nu ; \frac{x}{1-z+z x}, \frac{x z}{1-z+z x}\right] \text {. } \\
& \sum_{n=0}^{\infty} \frac{(\lambda)_{n}}{n !}{ }_{2} F_{1}\left[\begin{array}{c}
-n, \mu ; x \\
\nu ;
\end{array}\right]{ }_{r+1} F_{s}\left[\begin{array}{r}
-n, \alpha_{1}, \cdots, \alpha_{r} ; \\
1-\lambda-n, \beta_{1}, \cdots, \beta_{s}
\end{array} ;\right]^{n} \\
& =(1-z)^{\mu-\lambda}(1-z+z x)^{-\mu} \sum_{n=0}^{\infty} \frac{\left(\alpha_{1}\right)_{n} \cdots\left(\alpha_{r}\right)_{n}}{\left(\beta_{1}\right)_{n} \cdots\left(\beta_{s}\right)_{n}} \frac{(y z)^{n}}{n !} \\
& \cdot F_{1}\left[\mu,-n, \nu-\lambda+n ; \nu ; \frac{x}{1-z+z x}, \frac{x z}{1-z+z x}\right] \text {. }
\end{aligned}
$$




$$
\begin{aligned}
& \sum_{n=0}^{\infty} \frac{(\lambda)_{n}}{n !}{ }_{2} F_{1}\left[\begin{array}{c}
-n, \mu ; \\
\nu ;
\end{array}\right]{ }_{r+1} F_{s}\left[\begin{array}{r}
\lambda+n, \alpha_{1}, \cdots, \alpha_{r} ; \\
\beta_{1}, \cdots, \beta_{s}
\end{array} ; y\right] z^{n} \\
& =(1-z)^{\mu-\lambda}(1-z+z x)^{-\mu} \sum_{n=0}^{\infty} \frac{(\lambda)_{n}\left(\alpha_{1}\right)_{n} \cdots\left(\alpha_{r}\right)_{n}}{n !\left(\beta_{1}\right)_{n} \cdots\left(\beta_{s}\right)_{n}}\left(\frac{y}{1-z}\right)^{n} \\
& \cdot F_{1}\left[\mu,-n, \nu-\lambda ; \nu ; \frac{x}{1-z+z x}, \frac{x z}{1-z+z x}\right] \text {. } \\
& \sum_{n=0}^{\infty} \frac{(\lambda)_{n}}{n !}{ }_{2} F_{1}\left[\begin{array}{c}
\lambda+n, \mu ; \\
\nu
\end{array} ;\right]{ }_{r+1} F_{s}\left[\begin{array}{r}
\lambda+n, \alpha_{1}, \cdots, \alpha_{r} ; \\
\beta_{1}, \cdots, \beta_{s} ;
\end{array}\right] z^{n} \\
& =(1-z)^{\mu-\lambda}(1-z-x)^{-\mu} \sum_{n=0}^{\infty} \frac{(\lambda)_{n}\left(\alpha_{1}\right)_{n} \cdots\left(\alpha_{r}\right)_{n}}{n !\left(\beta_{1}\right)_{n} \cdots\left(\beta_{s}\right)_{n}}\left(\frac{y}{1-z}\right)^{n} \\
& \text { - } F_{1}\left[\mu,-n, \nu-\lambda ; \nu ; \frac{x z}{x+z-1}, \frac{x}{x+z-1}\right] \text {. }
\end{aligned}
$$

3. Further Generalizations. The second member of (2.7) can be written in the form

$$
\begin{aligned}
(1-z)^{\mu-\lambda} & (1-z+z x)^{-\mu} \sum_{n=0}^{\infty} \frac{(\lambda)_{n}\left(\alpha_{1}\right)_{n} \cdots\left(\alpha_{r}\right)_{n}}{\left(\beta_{1}\right)_{n} \cdots\left(\beta_{s}\right)_{n}}\left(\frac{y z}{z-1}\right)^{n} \\
& \cdot \sum_{m=0}^{n} \frac{(\mu)_{m}}{m !(n-m) !(\nu)_{m}}\left(-\frac{x}{1-z+z x}\right)^{m}{ }_{2} F_{1}\left[\begin{array}{r}
\nu-\lambda, \mu+m ; \\
\nu+m ; 1-z+z x
\end{array}\right]
\end{aligned}
$$

and since $[7$, p. 64]

$$
{ }_{2} F_{1}\left[\begin{array}{r}
a, b ; z \\
c ;
\end{array}\right]=(1-z)^{-b}{ }_{2} F_{1}\left[\begin{array}{r}
c-a, b ; \frac{z}{z-1} \\
c ;
\end{array}\right],
$$

this equals

$$
\begin{gathered}
(1-z)^{-\lambda} \sum_{n=0}^{\infty} \frac{(\lambda)_{n}\left(\alpha_{1}\right)_{n} \cdots\left(\alpha_{r}\right)_{n}}{\left(\beta_{1}\right)_{n} \cdots\left(\beta_{s}\right)_{n}}\left(\frac{y z}{z-1}\right)^{n} \\
\cdot \sum_{m=0}^{n} \frac{(\mu)_{m}}{m !(n-m) !(\nu)_{m}}\left(\frac{x}{z-1}\right)^{m}{ }_{2} F_{1}\left[\begin{array}{r}
\lambda+m, \mu+m ; \\
\nu+m ; z-1
\end{array}\right] \\
=(1-z)^{-\lambda} \sum_{m=0}^{\infty} \frac{(\lambda)_{m}(\mu)_{m}\left(\alpha_{1}\right)_{m} \cdots\left(\alpha_{r}\right)_{m}}{m !(\nu)_{m}\left(\beta_{1}\right)_{m} \cdots\left(\beta_{s}\right)_{m}}\left[\frac{x y z}{(1-z)^{2}}\right]^{m} \\
\cdot{ }_{2} F_{1}\left[\begin{array}{r}
\lambda+m, \mu+m ; \frac{x z}{\nu+m} \\
\nu+m-1
\end{array}\right]{ }_{r+1} F_{s}\left[\begin{array}{r}
\lambda+m, \alpha_{1}+m, \cdots, \alpha_{r}+m ; \\
\beta_{1}+m, \cdots, \beta_{s}+m ; z-1
\end{array}\right],
\end{gathered}
$$

which leads us to

$$
\begin{aligned}
& \sum_{n=0}^{\infty} \frac{(\lambda)_{n}}{n !}{ }_{2} F_{1}\left[\begin{array}{r}
-n, \mu ; x \\
\nu ;
\end{array}\right]{ }_{r+1} F_{s}\left[\begin{array}{r}
-n, \alpha_{1}, \cdots, \alpha_{r} ; \\
\beta_{1}, \cdots, \beta_{s}
\end{array} ; y\right] z^{n} \\
& =(1-z)^{-\lambda} \sum_{n=0}^{\infty} \frac{(\lambda)_{n}(\mu)_{n}\left(\alpha_{1}\right)_{n} \cdots\left(\alpha_{r}\right)_{n}}{n !(\nu)_{n}\left(\beta_{1}\right)_{n} \cdots\left(\beta_{s}\right)_{n}}\left[\frac{x y z}{(1-z)^{2}}\right]^{n}
\end{aligned}
$$

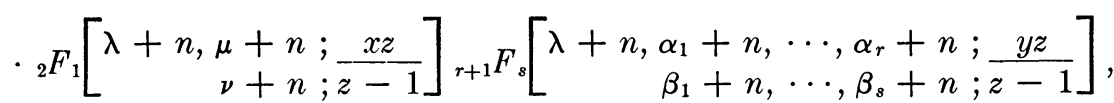


where $r \leqq s$ and $|z|$ is sufficiently small.

Starting from (3.1) and making use of the foregoing operational techniques it is readily seen by induction that

$$
\begin{aligned}
& \sum_{n=0}^{\infty} \frac{(\lambda)_{n}}{n !}{ }_{p+1} F_{q}\left[\begin{array}{r}
-n, a_{1}, \cdots, a_{p} ; \\
b_{1}, \cdots, b_{q} ;
\end{array}\right]{ }_{r+1} F_{s}\left[\begin{array}{r}
-n, \alpha_{1}, \cdots, \alpha_{r} ; \\
\beta_{1}, \cdots, \beta_{s} ;
\end{array}\right] z^{n} \\
& =(1-z)^{-\lambda} \sum_{n=0}^{\infty} \frac{(\lambda)_{n}\left(a_{1}\right)_{n} \cdots\left(a_{p}\right)_{n}\left(\alpha_{1}\right)_{n} \cdots\left(\alpha_{r}\right)_{n}}{n !\left(b_{1}\right)_{n} \cdots\left(b_{q}\right)_{n}\left(\beta_{1}\right)_{n} \cdots\left(\beta_{s}\right)_{n}}\left[\frac{x y z}{(1-z)^{2}}\right]^{n} \\
& \cdot{ }_{p+1} F_{q}\left[\begin{array}{r}
\lambda+n, a_{1}+n, \cdots, a_{p}+n ; \frac{x z}{z-1} \\
b_{1}+n, \cdots, b_{q}+n ; z-1,
\end{array}\right] \\
& \cdot{ }_{r+1} F_{s}\left[\begin{array}{r}
\lambda+n, \alpha_{1}+n, \cdots, \alpha_{r}+n ; y z \\
\beta_{1}+n, \cdots, \beta_{s}+n ; z-1
\end{array}\right],
\end{aligned}
$$

provided $p \leqq q, r \leqq s$ and $|x|,|y|$, and $|z|$ are so constrained that the two sides have a meaning.

In a similar manner we have

$$
\begin{aligned}
& \sum_{n=0}^{\infty} \frac{(\lambda)_{n}}{n !}{ }_{p+1} F_{q}\left[\begin{array}{r}
-n, a_{1}, \cdots, a_{p} ; \\
b_{1}, \cdots, b_{q} ;
\end{array}\right]{ }_{r+1} F_{s+1}\left[\begin{array}{r}
-n, \alpha_{1}, \cdots, \alpha_{r} ; \\
1-\lambda-n, \beta_{1}, \cdots, \beta_{s} ;
\end{array}\right] z^{n} \\
& =(1-z)^{-\lambda} \sum_{n=0}^{\infty} \frac{\left(a_{1}\right)_{n} \cdots\left(a_{p}\right)_{n}\left(\alpha_{1}\right)_{n} \cdots\left(\alpha_{r}\right)_{n}}{\left(b_{1}\right)_{n} \cdots\left(b_{q}\right)_{n}\left(\beta_{1}\right)_{n} \cdots\left(\beta_{s}\right)_{n}} \frac{(-x y z)^{n}}{n !} \\
& \cdot{ }_{p+1} F_{q}\left[\begin{array}{r}
\lambda, a_{1}+n, \cdots, a_{p}+n ; \frac{x z}{} \\
b_{1}+n, \cdots, b_{q}+n ; z-1
\end{array}\right]{ }_{r} F_{s}\left[\begin{array}{c}
\alpha_{1}+n, \cdots, \alpha_{r}+n ; y z \\
\beta_{1}+n, \cdots, \beta_{s}+n ; y
\end{array}\right], \\
& \sum_{n=0}^{\infty} \frac{(\lambda)_{n}}{n !}{ }_{p+1} F_{q}\left[\begin{array}{r}
-n, a_{1}, \cdots, a_{p} ; \\
b_{1}, \cdots, b_{q} ; x
\end{array}\right]{ }_{r+1} F_{s}\left[\begin{array}{r}
\lambda+n, \alpha_{1}, \cdots, \alpha_{r} ; \\
\beta_{1}, \cdots, \beta_{s} ;
\end{array}\right] z^{n} \\
& =(1-z)^{-\lambda} \sum_{n=0}^{\infty} \frac{(\lambda)_{n}\left(a_{1}\right)_{n} \cdots\left(a_{p}\right)_{n}\left(\alpha_{1}\right)_{n} \cdots\left(\alpha_{r}\right)_{n}}{n !\left(b_{1}\right)_{n} \cdots\left(b_{q}\right)_{n}\left(\beta_{1}\right)_{n} \cdots\left(\beta_{s}\right)_{n}}\left[-\frac{x y z}{(1-z)^{2}}\right]^{n} \\
& \cdot{ }_{p+1} F_{q}\left[\begin{array}{r}
\lambda+n, a_{1}+n, \cdots, a_{p}+n ; x z \\
b_{1}+n, \cdots, b_{q}+n ; z-1
\end{array}\right] \\
& \cdot{ }_{r+1} F_{s}\left[\begin{array}{r}
\lambda+n, \alpha_{1}+n, \cdots, \alpha_{r}+n ; y \\
\beta_{1}+n, \cdots, \beta_{s}+n ; 1-z
\end{array}\right]
\end{aligned}
$$

and

$$
\begin{aligned}
& \sum_{n=0}^{\infty} \frac{(\lambda)_{n}}{n !}{ }_{p+1} F_{q}\left[\begin{array}{r}
\lambda+n, a_{1}, \cdots, a_{p} ; \\
b_{1}, \cdots, b_{q} ;
\end{array}\right]{ }_{r+1} F_{s}\left[\begin{array}{r}
\lambda+n, \alpha_{1}, \cdots, \alpha_{r} ; \\
\beta_{1}, \cdots, \beta_{s} ;
\end{array}\right] z^{n} \\
& =(1-z)^{-\lambda} \sum_{n=0}^{\infty} \frac{(\lambda)_{n}\left(a_{1}\right)_{n} \cdots\left(a_{p}\right)_{n}\left(\alpha_{1}\right)_{n} \cdots\left(\alpha_{r}\right)_{n}}{n !\left(b_{1}\right)_{n} \cdots\left(b_{q}\right)_{n}\left(\beta_{1}\right)_{n} \cdots\left(\beta_{s}\right)_{n}}\left[\frac{x y z}{(1-z)^{2}}\right]^{n}
\end{aligned}
$$

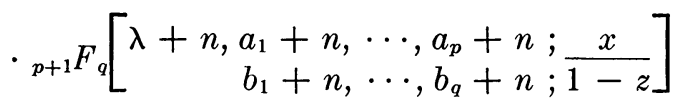

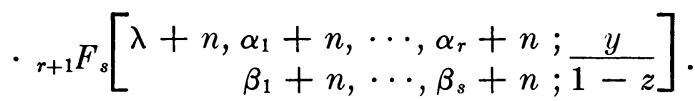


All these bilinear relations are valid for such values of $|x|,|y|$, and $|z|$ that the two sides do possess a meaning, provided $p \leqq q$, and $r \leqq s$ except possibly in (3.3) where $r$ cannot exceed $s+1$.

The special case $p=q=1$ of (3.2) corresponds to the formula (4), p. 1320 of Brafman [4] when $\lambda=b_{1}$, while in the limit as $x \rightarrow 0$ our formulas (2.7), (2.8) and (2.9) reduce to the results proved earlier by Chaundy [5, p. 62]. For $p=q=r=$ $s=1$, the bilinear relations (3.2), (3.4) and (3.5) are due to Meixner [12] who obtained them by transforming the Pochhammer contour integral associated with Gauss's hypergeometric function. The linear and bilinear generating functions discussed recently in [1] through [3] and in [13], [14], and [16] follow from our results in a straightforward manner.

Finally, we observe that in terms of the Humbert function [7, p. 225]

$$
\Phi_{3}[\alpha ; \beta ; x, y]=\sum_{m, n=0}^{\infty} \frac{(\alpha)_{m}}{(\beta)_{m+n}} \frac{x^{m}}{m !} \frac{y^{n}}{n !},
$$

the special case $p=r=s=0, q=1, b_{1}=1+\alpha, \lambda=-\beta$ of our result (3.3) gives us the interesting formula

$$
\begin{aligned}
& \sum_{n=0}^{\infty}\left(\begin{array}{c}
\alpha+n \\
n
\end{array}\right)^{-1} L_{n}^{(\alpha)}(x) L_{n}{ }^{(\beta-n)}(y) z^{n} \\
& \quad=(1+z)^{\beta} \exp (-y z) \Phi_{3}\left[-\beta ; 1+\alpha ; \frac{x z}{1+z}, x y z\right]
\end{aligned}
$$

which reduces to the known generating relation [8, p. 215]

$$
\begin{aligned}
& \sum_{n=0}^{\infty} \frac{(-)^{n} n !}{\Gamma(\alpha+n+1)} L_{n}^{(\alpha)}(x) L_{n}^{(\beta-n)}(y) \\
& \quad=x^{-(\alpha-\beta) / 2} y^{-(\alpha+\beta) / 2} \exp (y) J_{\alpha+\beta}\left[2(x y)^{1 / 2}\right]
\end{aligned}
$$

when $z \rightarrow-1$.

West Virginia University

Morgantown, West Virginia 26506

1. N. Abdul-Halim \& W. A. Al-Salam, "Double Euler transformations of certain hypergeometric functions," Duke Math. J., v. 30, 1963, pp. 51-62. MR 26 \#1500.

2. F. Brafman, "Generating functions of Jacobi and related polynomials," Proc. Amer. Math. Soc., v. 2, 1951, pp. 942-949. MR 13,649.

3. F. BRAFMAN, "Some generating functions for Laguerre and Hermite polynomials," Canad. J. Math., v. 9, 1957, pp. 180-187. MR 19, 28.

4. F. BRAFMAN, "An ultraspherical generating function," Pacific J. Math., v. 7, 1957, pp. 1319-1323. MR $21 \# 1410$.

5. T. W. CHAUNDY, "An extension of hypergeometric functions," Quart. J. Math. Oxford Ser., v. 14, 1943, pp. 55-78. MR 6, 64.

6. A. ERDÉLYI, "Funktionalrelationen mit konfluenten hypergeometrischen Funktionen. II: Reihenentwicklungen," Math. Z., v. 42, 1937, pp. 641-670.

7. A. ERDÉLYI, et al., Higher Transcendental Functions. Vol. I, McGraw-Hill, New York, 1953. MR 15, 419.

8. A. ERDÉLYI, et al., Higher Transcendental Functions, Vol. II, McGraw-Hill, New York, 1953. MR 15, 419 .

9. A. ERdÉLYi, et al., Tables of Integral Transforms, Vol. I, McGraw-Hill, New York, 1954. MR 15, 868 .

10. G. H. HARDy, "Summation of a series of polynomials of Laguerre," J. London Math. Soc., v. 7, 1932, pp. 138-139; addendum, 192. 
11. E. Hille, "On Laguerre's series. I, II, III," Proc. Nat. Acad. Sci. U.S.A., v. 12, 1926, pp. 261-269; 348-30̃2.

12. J. Meixner, "Umformung gewisser Reihen, deren Glieder Produkte hypergeometrischer Funktionen sind," Deutsche Math., v. 6, 1942, pp. 341-349. MR 4, 275.

13. H. M. Srivastava \& C. M. Joshi, "Certain double Whittaker transforms of generalized hypergeometric functions," Yokohama Math. J., v. 15, 1967, pp. 17-32.

14. H. M. SRivastava \& J. P. Singhal, "Double Meijer transformations of certain hypergeometric functions," Proc. Cambridge Philos. Soc., v. 64, 1968, pp. 425-430. MR 36 \#6660.

15. G. N. WATSON, "Notes on generating functions of polynomials. I: Laguerre polynomials," J. London Math. Soc., v. 8, 1933, pp. 189-192.

16. L. WEISNER, "Group-theoretic origin of certain generating functions," Pacific J. Math.. v. 5, 1955, pp. 1033-1039. MR 19, 264. 